\title{
ON A WEAK-TYPE THEOREM WITH APPLICATIONS
}

\author{
By LARS ERIK PERSSON
}

[Received 14 October 1976]

\section{Introduction}

We assume that $f$ is a measurable, complex-valued function on a measure space $(\Omega, \mu)$. The function $|f|$ can be rearranged to become a nonincreasing function, $f^{*}$, on $[0, \infty[$, which is equidistributed with $|f|$ and continuous from the right (see, for example, [5, pp. 249-51]). The Lorentz space $\Lambda(\lambda, q)(\lambda=\lambda(t) \geqslant 0,0<t<\infty$, and $q>0)$ is the collection of all $f$ such that $\|f\|_{\lambda, q}^{*}<\infty$, where

$$
\|f\|_{\lambda, q}^{*}= \begin{cases}\left(\int_{0}^{\infty}\left(\left(f^{*}(t)\right) \lambda(t)\right)^{q} \frac{d t}{t}\right)^{1 / q}, & \text { for } q<\infty, \\ \sup _{b>0} f^{*}(t) \lambda(t), & \text { for } q=\infty\end{cases}
$$

(see [8, p. 37]). We note that if $\lambda(t)=t^{1 / p}$ then $\|f\|_{\lambda, q}^{*}$ is just the usual $L(p, q)$-norm $\|f\|_{p, q}^{*}$.

We say that $f$ belongs to the average space $\Lambda_{A}(\lambda, q, p, r)(\lambda=\lambda(t) \geqslant 0$, $0<q<\infty, 0<p<\infty$, and $0<r<\infty)$ if

$$
\|f\|_{\lambda, q, p, r}^{* *}=\left(\int_{0}^{K}(\lambda(t))^{q_{t}-q / p}\left(\int_{0}^{t}\left(f^{*}(u)\right)^{r} u^{r / p} \frac{d u}{u}\right)^{q / r} \frac{d t}{t}\right)^{1 / q}<\infty,
$$

where $K=\sup \left\{t \mid f^{*}(t)>0\right\}$. (We do not exclude the case where $K=\infty$.) For the case where $p=r$ we write $\|f\|_{\lambda, q, p, r}^{* *}=\|f\|_{\lambda, q, r}^{* *}$ and $f \in \Lambda_{A}(\lambda, q, r)$ when this quantity is finite.

Moreover, we shall say that a non-negative function $\lambda$ on $] 0, \infty[$ belongs to the space $Q\left(a_{0}, b_{0}\right)$, if, for some $a<a_{0}$, and $b>b_{0}, \lambda(t) t a$ is an increasing, and $\lambda(t) t^{b}$ is a decreasing, function of $t$ (see [11 and 12]). Sometimes we want to say that $\lambda \in Q\left(a_{0}, b\right)$ for some real number $b$ and then we write $\lambda \in Q\left(a_{0},-\right)$.

In [11] the present author has stated

Theorem I. Let $0<q<\infty$, let $f \in L[0,1]$, and let $c_{n}$, for $n \in \mathbf{Z}$, be the complex Fourier coefficients of $f$.

(a) If $\lambda \in Q\left(-\frac{1}{2},-\right)$, then

$$
\left(\sum_{n=1}^{\infty}\left(c_{n}^{*}\right)^{q}\left(n \lambda\left(n^{-1}\right)\right)^{q} n^{-1}\right)^{1 / q} \leqslant A\|f\|_{\lambda, q, 1}^{* *},
$$

where $A$ depends on $\lambda$ and $q$ only. 
(b) If $\lambda \in Q\left(-\frac{1}{2},-1\right)$, then (1.1) holds with $\|f\|_{\lambda, q, 1}^{* *}$ replaced by $\|f\|_{\lambda, q}^{*}$.

As usual, the sequence $\left(\left.c_{n}^{*}\right|_{0} ^{\infty}\right.$ is the sequence $\left(\left|c_{n}\right|\right)_{-\infty}^{\infty}$ rearranged in non-increasing order. We note that Theorem I can be seen as a generalization and a refinement of well-known results of Hardy and Littlewood, and Stein (see [14, p. 490] and [17, vol. II, p. 123]), and Zygmund and Bennett (see [1, p. 3] and [17, vol. II, p. 158]). The obvious dual version of Theorem I and complementary examples can also be found in [11].

The proof of Theorem I depends crucially upon the facts that the Fourier operator $F_{d}(f)=(\hat{f}(n))_{-\infty}^{\infty}=\left(c_{n}\right)_{-\infty}^{\infty}$ is a linear mapping of (weak-) type $(2,2)$ (Parseval's relation) and that $\left|c_{n}\right| \leqslant \int_{0}^{1}|f(t)| d t$, where $n \in \mathbf{Z}$. In this paper we shall generalize Theorem I so that the theorem can be applied to more general quasi-linear operators $T$. As usual, we shall say that an operator $T$ is quasi-linear if $T(f+g)$ is defined whenever $T(f)$ and $T(g)$ are defined and if $|T(f+g)| \leqslant A(|T f|+|T g|)$, where $A$ is a constant which does not depend on $f$ and $g$. For such operators we have the following useful estimate:

$$
(T(f+g))^{*}(2 t) \leqslant A\left((T f)^{*}(t)+(T g)^{*}(t)\right) \quad(t>0)
$$

(see [5, p. 263]). It is well known that many classical operators (for example, the Hilbert transform, the Fourier transform, fractional integral operators, singular integral operators, and so on) map $L(p, q)$ boundedly into some $L\left(p_{1}, q\right)$ (see, for example, [5 and 16]). In particular, the results in this paper will show that the same operators even map $\Lambda(\lambda, q)$ boundedly into $\Lambda\left(\lambda_{1}, q\right)$ ( $\lambda$ and $\lambda_{1}=\lambda_{1}(\lambda)$ belong to certain $Q$-classes).

The main results in this paper can be found in $\S 2$. In particular, Theorem 2.1 can be seen as a refinement of a well-known interpolation theorem of Marcinkiewicz, Calderón, and Hunt (see [5, p. 264]). The proofs of the main results are carried out in $\$ 3$. Some applications and relations to well-known results are pointed out in $\$ 4$.

\section{The main results}

We shall use the following notation:

and

$$
\begin{aligned}
K & =\sup \left\{t \mid f^{*}(t)>0\right\} ; \\
\gamma & =\left(\frac{1}{p_{1}}-\frac{1}{p_{0}}\right) /\left(\frac{1}{p_{1}^{\prime}}-\frac{1}{p_{0}^{\prime}}\right) ; \\
I & =I(K, \gamma)= \begin{cases}{\left[0,2 K^{\gamma}\right],} & \text { for } \gamma>0, \\
{\left[2 K^{\gamma}, \infty[,\right.} & \text { for } \gamma<0 ;\end{cases}
\end{aligned}
$$

$$
\lambda_{1}=\lambda_{1}(t, \lambda, \gamma)=t^{\left.\left(\left(1 / p_{1} p_{0}{ }^{\prime}\right)-\left(1 / p_{1} p_{0}\right)\right) /\left(1 / p_{1}\right)-\left(1 / p_{0}\right)\right)} \lambda\left(t^{1 / \gamma}\right) .
$$


TheOREM 2.1. Let $q, p_{i}, q_{i}, p_{i}^{\prime}, q_{i}^{\prime}$, where $i=0,1$, be real numbers such that $0<q \leqslant \infty, \quad 0<p_{0}<p_{1} \leqslant \infty, \quad 0<q_{0}<\infty, \quad 0<q_{1} \leqslant \infty \quad\left(p_{1}=\infty\right.$ when $\left.q_{1}=\infty\right)$, and $p_{0}^{\prime} \neq p_{1}^{\prime}$. Moreover, let $\lambda \in Q\left(-1 / p_{1},-1 / p_{0}\right)$ and let $T$ be a quasi-linear operator such that

$$
\|T f\|_{p_{i}, q_{i}}^{*} \leqslant A_{i}\|f\|_{p_{i}, q_{i}}^{*}(i=0,1) .
$$

Then, for every $s \geqslant q$,

$$
\|T f\|_{\lambda_{1}, s}^{*} \leqslant A\|f\|_{\lambda, q}^{*} .
$$

The constant $A$ depends only on $\lambda, q, A_{i}, p_{i}$, and $q_{i}$.

We note that if

$$
\frac{1}{p_{\theta}}=\frac{1-\theta}{p_{0}}+\frac{\theta}{p_{1}}, \quad \frac{1}{p_{\theta}^{\prime}}=\frac{1-\theta}{p_{0}^{\prime}}+\frac{\theta}{p_{1}^{\prime}} \quad(0<\theta<1),
$$

and if $\lambda(t)=t^{1 / p_{\theta}}$, then $\lambda(t) \in Q\left(-1 / p_{1},-1 / p_{0}\right)$ and $\lambda_{1}(t)=t^{1 / p_{\theta^{\prime}}}$. Therefore, for every $s \geqslant q$,

$$
\|T f\|_{p_{\theta^{\prime}, s}}^{*} \leqslant A\|f\|_{p^{\prime} q}^{*}
$$

and this is just a well-known interpolation estimate of Marcinkiewicz, Calderón, and Hunt [5, p. 264]. We can even state the following corollary.

Corollary 2.2. Let $a \in R$, let $T, q, p_{i}, q_{i}, p_{i}^{\prime}, q_{i}^{\prime}, p_{\theta}$, and $p_{\theta}^{\prime}$ be defined as above, and let $f \in \Lambda\left(t^{1 / p_{\theta}}(\log (2+t))^{a}, q\right)$. Then

$$
T f \in \Lambda\left(t^{1 / p_{\theta^{\prime}}}\left(\log \left(2+t^{\mathrm{ggn}\left(p_{1}-p_{0}{ }^{\prime}\right)}\right)\right)^{a}, q\right)
$$

(where $\operatorname{sgn} b=b /|b|$ ).

The proof of the corollary depends on the fact that, for every $b \neq 0$, there exist positive constants $A_{i}(i=0,1)$, such that

$$
A_{0} \log \left(2+t^{\mathrm{ggn} b}\right) \leqslant \log \left(2+t^{b}\right) \leqslant A_{1} \log \left(2+t^{\mathrm{ggn} b}\right) .
$$

Therefore we find that if $\lambda(t)=t^{1 / p_{\theta}}(\log (2+t))^{a}$, then

$$
\lambda_{1}(t) \simeq t^{1 / p_{\theta^{\prime}}}\left(\log \left(2+t^{\operatorname{sgn}\left(p_{1}^{\prime}-p_{0^{\prime}}\right)}\right)\right)^{a} .
$$

(We can, for example, choose $A_{0}=\frac{1}{2} \min (|b|, 1)$ and $A_{1}=2 \log \left(2+2^{|b|}\right)$.)

It is easy to find examples to show that (2.2) does not hold, in general, for the case where $\theta=0$. However, it is possible to make a similar estimate which does not exclude this and other exceptional cases.

Theorem 2.3. Assume that $p_{i}, q_{i}, p_{i}^{\prime}, q_{i}^{\prime}(i=0,1)$ are positive numbers such that $q_{i}<\infty, p_{0}<p_{1}<\infty$, and $p_{0}^{\prime} \neq p_{1}^{\prime}$. If $T$ is a quasi-linear operator satisfying (2.1) and if $\lambda \in Q\left(-1 / p_{1},-\right)$, then, for every $q$, with $0<q<\infty$,

$$
\int_{I}\left((T f)^{*}(t)\left(\lambda_{1}(t)\right)\right)^{q} \frac{d t}{t} \leqslant A\left(\|f\|_{\lambda, q, p_{0}, q_{0}}^{* *}\right)^{q}
$$

where the constant $A$ depends at most on $\lambda, q, A_{i}, p_{i}$, and $q_{i}$. 
The proofs of Theorems 2.1 and 2.3 depend crucially upon some estimates, which we shall state separately as lemmas. Our first lemma is used to estimate the functionals

$$
L(t, f)=t^{-1 / p_{0}}\left(\int_{0}^{1^{1 / v}}\left(f^{*}(u)\right)^{q_{0}} u^{q_{0} / p_{0}} \frac{d u}{u}\right)^{1 / a_{0}}+t^{-1 / p^{\prime}}\left(\int_{l^{1 / \gamma}}^{\infty}\left(f^{*}(u)\right)^{q_{1}} u^{q_{1} / p_{1}} \frac{d u}{u}\right)^{1 / q_{1}} .
$$

Leman 2.4. Assume that $p_{i}, q_{i}, p_{i}^{\prime}, q_{i}^{\prime}(i=0,1)$ are positive numbers such that $q_{i}<\infty, p_{0}<p_{1}<\infty$, and $p_{0}^{\prime} \neq p_{1}^{\prime}$. If $T$ is a quasi-linear operator satisfying (2.1), then, for $t>0$,

$$
(T f)^{*}(2 t) \leqslant A L(t, f),
$$

where $A$ depends only on $A_{i}, p_{i}$, and $q_{i}$.

The next lemma can be seen as a generalization of a well-known estimate of Hardy (see, for example, [17, vol. I, p. 20]).

LEMMA 2.5. Let $\lambda$ and $\psi$ be non-negative functions on $[0, \infty[$ such that $\psi(u) \leqslant A_{0} \psi(t)$ for some $A_{0} \in \mathbf{R}^{+}$and for $u \in[t, 2 t](t>0)$. Moreover, let $0<q<\infty, 0<r<\infty$, and put $\varphi_{q}(t)=(\lambda(t))^{q}(\psi(t))^{-q} t^{-1}$.

(a) If $\varphi_{Q} \in Q(-, 1)$, then

$$
\left(\int_{0}^{\infty} \varphi_{u}(t)\left(\int_{0}^{t}\left(\left(f^{*}(u)\right)(\psi(u))\right)^{r} \frac{d u}{u}\right)^{q / r} d t\right)^{1 / q} \leqslant A\|f\|_{\lambda q^{*}}^{*}
$$

(b) If $\varphi_{Q} \in Q(1,-)$, then

$$
\left(\int_{0}^{\infty} \varphi_{u}(t)\left(\int_{t}^{\infty}\left(\left(f^{*}(u)\right)(\psi(u))\right)^{r} \frac{d u}{u}\right)^{q / r} d t\right)^{1 / q} \leqslant A\|f\|_{\lambda q^{*}}^{*}
$$

The constant $A$ depends only on $\lambda, \psi$, and $q$.

3. Proofs

Proof of Lemma 2.4. We put

$$
\begin{gathered}
f_{y}(x)= \begin{cases}f(x) & \text { if }|f(x)| \leqslant y, \\
y \operatorname{sgn} f(x) & \text { if }|f(x)|>y,\end{cases} \\
f^{\nu}(x)=f(x)-f_{y}(x), \\
g_{1}=T f^{y} \text { and } g_{2}=T f_{y} .
\end{gathered}
$$

For each $t$ we choose $y=y(t)=f^{*}\left(t^{1 / \gamma}\right)$ and note that

$$
\left(f_{y}\right)^{*}(u)= \begin{cases}y & \text { if } 0<u \leqslant t^{1 / \gamma} \\ f^{*}(u) & \text { if } u>t^{1 / \gamma}\end{cases}
$$

$\left(f^{\nu}\right)^{*}(u)=0$ for $u>t^{1 / \gamma}$ and $\left(f^{\nu}\right)^{*}(u) \leqslant f^{*}(u)$ for $0<u \leqslant t^{1 / \gamma}$. Since $\|f\|_{p, r_{1}}^{*} \leqslant\|f\|_{p, n_{n}}^{*}$ for $r_{2} \leqslant r_{1}$ (see [5, p. 253]), we can, without loss of generality, assume that $q_{0}^{\prime}=q_{1}^{\prime}=\infty$ in (2.1). Therefore when we apply 
(2.1) (with $i=0$ ) to the function $f^{\nu}(x)$ we find that, for each $t>0$,

$$
\begin{aligned}
g_{1}^{*}(t) t^{1 / p_{0}} & \leqslant A_{0}\left\|f^{\nu}\right\|_{p_{0}, q_{0}} \\
& \leqslant A_{0}\left(\int_{0}^{\left(t^{1 / \gamma}\right.}\left(f^{*}(u)\right)^{q_{0} u^{q_{0} / p_{0}}} \frac{d u}{u}\right)^{1 / q_{0}}=A_{0} I_{1}^{1 / q_{0}} .
\end{aligned}
$$

Analogously, by (2.1) (with $i=1$ ),

$$
\begin{aligned}
g_{2}^{*}(t) t^{1 / p_{1}} & \leqslant A_{1}\left\|f_{y}\right\|_{p_{1}, q_{1}} \\
& =A_{1}\left(\int_{0}^{t^{1 / \gamma}} y^{a_{1} u^{q_{1} / p_{1}}} \frac{d u}{u}+\int_{t^{1 / \gamma}}^{\infty}\left(f^{*}(u)\right)^{\alpha_{1}} u^{q_{1} / p_{1}} \frac{d u}{u}\right)^{1 / \alpha_{1}} \\
& =A_{1}\left(I_{2}+I_{3}\right)^{1 / \alpha_{1}} .
\end{aligned}
$$

Therefore, by the inequality

$$
(a+b)^{\beta} \leqslant \max \left(2^{\beta-1}, 1\right)\left(a^{\beta}+b^{\beta}\right) \quad(a>0, b>0, \beta>0),
$$

we find that, for each $t>0$,

Also

$$
g_{2}^{*}(t) t^{1 / p_{1}} \leqslant A_{2}\left(I_{2}^{1 / q_{1}}+I_{3}^{1 / q_{1}}\right) .
$$

$$
\begin{aligned}
I_{2}^{1 / q_{1}} & =\left(\int_{0}^{t^{1 / \gamma}} y^{a_{1}} u^{\alpha_{1} / p_{1}} \frac{d u}{u}\right)^{1 / \alpha_{1}} \\
& =A_{3} f^{*}\left(t^{1 / \gamma}\right) t^{1 / \gamma p_{1}} \\
& \leqslant A_{4} t^{\left(1 / \gamma p_{1}\right)-\left(1 / \gamma p_{0}\right)}\left(\int_{0}^{t^{1 / \gamma}}\left(f^{*}(u)\right)^{\alpha_{0}} u^{q_{0} / p_{0}} \frac{d u}{u}\right)^{1 / q_{0}} \\
& =A_{4} t^{\left(1 / \gamma p_{1}\right)-\left(1 / \gamma p_{0}\right)} I_{1}^{1 / q_{0}} .
\end{aligned}
$$

Thus, according to (1.2), the definition of $\gamma$, and (3.1)-(3.4),

$$
\begin{aligned}
(T f)^{*}(2 t) & \leqslant A_{5}\left(t^{\left(1 / \gamma p_{1}\right)-\left(1 / \gamma p_{0}\right)-\left(1 / p_{1}{ }^{\prime}\right)}+t^{-1 / p_{0}{ }^{\prime}}\right) I_{1}^{1 / q_{0}}+A_{2} t^{-1 / p_{1}} I_{3}^{1 / q_{1}} \\
& \leqslant A\left(t^{-1 / p_{0}{ }^{\prime}} I_{1}^{1 / q_{0}}+t^{-1 / p_{1}^{\prime}} I_{3}^{1 / q_{1}}\right)=A L(t, f) .
\end{aligned}
$$

The proof is complete.

The proof of Lemma 2.5 is based upon the following corresponding result for sequences:

LEMma. Let $\left(a_{k}\right)_{n_{0}}^{n_{1}}$ and $\left(b_{k}\right)_{n_{0}}^{n_{1}}$ be sequences where $a_{k}>0$ and $b_{k} \geqslant 0$ for every $k$, and $\left|n_{0}\right|+\left|n_{1}\right|=\infty$. Then, for $d \geqslant 1$,

$$
\sum_{k=n_{0}}^{k=n_{1}} a_{k}\left(\sum_{n=n_{0}}^{n=k} b_{n}\right)^{d} \leqslant d^{d} \sum_{k=n_{0}}^{k-n_{1}}\left(a_{k}\right)^{1-d}\left(\sum_{n=k}^{n=n_{1}} a_{n}\right)^{d}\left(b_{k}\right)^{d}
$$

and

$$
\sum_{k=n_{0}}^{k=n_{1}} a_{k}\left(\sum_{n=k}^{n-n_{1}} b_{n}\right)^{d} \leqslant d^{d} \sum_{k=n_{0}}^{k=n_{1}}\left(a_{k}\right)^{1-d}\left(\sum_{n=n_{0}}^{n-k} a_{n}\right)^{d}\left(b_{k}\right)^{d} .
$$

The constant $d^{d}$ is the best possible. 
Proof. For the case where $n_{0}=1$ and $n_{1}=\infty$, the lemma has been proved by Leindler [7, p. 279]. The proof of this more general case can be carried out in a similar way, so we omit the details.

Proof of Lemma 2.5. We can make some straightforward calculations to see that the condition ' $\varphi_{q} \in Q(-, 1)$ ' implies that, for every $x>0$,

$$
0<\int_{x}^{\infty} \varphi_{u}(t) d t \leqslant B_{1} \int_{x}^{2 x} \varphi_{u}(t) d t \leqslant B_{2} \int_{x / 2}^{x} \varphi_{y}(t) d t .
$$

Therefore, by applying the estimate (3.5) in the lemma above with $n_{0}=-\infty, n_{1}=\infty$,

$$
a_{k}=\int_{2^{k}}^{2^{k+1}} \varphi_{u}(t) d t \quad \text { and } \quad b_{k}=\int_{2^{k}}^{2^{k+1}}\left(\left(f^{*}(u)\right)(\psi(u))\right)^{r} \frac{d u}{u}
$$

we obtain

$$
\begin{aligned}
\int_{0}^{\infty} \varphi_{u}(t) & \left(\int_{0}^{l}\left(\left(f^{*}(u)\right)(\psi(u))\right)^{r} \frac{d u}{u}\right)^{q / r} d t \\
\leqslant & \sum_{-\infty}^{\infty} \int_{2^{k}}^{2^{k+1}} \varphi_{q}(t) d t\left(\sum_{-\infty}^{k} \int_{2^{n}}^{2^{n+1}}\left(\left(f^{*}(u)\right)(\psi(u))\right)^{r} \frac{d u}{u}\right)^{q / r} \\
& =\sum_{-\infty}^{\infty} a_{k}\left(\sum_{-\infty}^{k} b_{n}\right)^{q / r} \leqslant B_{3} \sum_{-\infty}^{\infty} a_{k} b_{k}^{q / r} .
\end{aligned}
$$

(For the case where $q<r$ we must make the trivial estimate

$$
\left(\sum_{-\infty}^{k} b_{n}\right)^{q / r} \leqslant \sum_{-\infty}^{k} b_{n}^{q / r}
$$

before we apply (3.5) with $d=1$.) Furthermore, by the growth conditions on $\psi$ and (3.7),

$$
\begin{aligned}
\sum_{-\infty}^{\infty} a_{k} b_{k}^{q / r} & =\sum_{-\infty}^{\infty} \int_{2^{k}}^{2^{k+1}} \varphi_{q}(t) d t\left(\int_{2^{k}}^{2^{k+1}}\left(\left(f^{*}(u)\right)(\psi(u))\right)^{r} \frac{d u}{u}\right)^{q / r} \\
& \leqslant B_{4} \sum_{-\infty}^{\infty} \int_{2^{k-1}}^{2^{k}} \varphi_{q}(t) d t \cdot\left(f^{*}\left(2^{k}\right)\right)^{q}\left(\psi\left(2^{k}\right)\right)^{q} \\
& \leqslant B_{5} \sum_{-\infty}^{\infty} \int_{2^{k-1}}^{2^{k}} \varphi_{q}(t)\left(f^{*}(t)\right)^{q}(\psi(t))^{q} d t \\
& =B_{5}\left(\|f\|_{\lambda, q}^{*}\right)^{q} .
\end{aligned}
$$

The proof of (a) follows when we combine this estimate with (3.8).

Analogously, it is easily seen that if $\varphi_{q} \in Q(1,-)$, then, for every $x>0$,

$$
0<\int_{0}^{2 x} \varphi_{q}(t) d t \leqslant B_{6} \int_{x}^{2 x} \varphi_{q}(t) d t \leqslant B_{7} \int_{x / 2}^{x} \varphi_{q}(t) d t .
$$


Therefore we can use (3.6) and make calculations similar to those above to prove part (b) of the lemma.

Proof of Theorem 2.3. According to Lemma 2.4 and (3.2) we see that for every $L \geqslant 0$,

$$
\begin{aligned}
& \int_{0}^{L}\left((T f)^{*}\left(2 t^{\prime}\right)\right)^{q} t^{q\left(\left(\gamma / p_{i}\right)-\left(1 / p_{i}\right)\right)}(\lambda(t))^{q} \frac{d t}{t} \\
& \leqslant B_{0}\left(\int_{0}^{L}(\lambda(t))^{q} t^{-q / p_{0}}\left(\int_{0}^{l}\left(f^{*}(u)\right)^{q_{0}} u^{q_{0} / p_{0}} \frac{d u}{u}\right)^{q / q_{0}} \frac{d t}{t}\right. \\
& \left.\quad+\int_{0}^{L}(\lambda(t))^{q} t^{-q / p_{1}}\left(\int_{t}^{\infty}\left(f^{*}(u)\right)^{q_{1}} u^{q_{1} / p_{1}} \frac{d u}{u}\right)^{q / q_{1}} \frac{d t}{t}\right) .
\end{aligned}
$$

The hypothesis $\lambda \in Q\left(-1 / p_{1},-\right)$ implies that

$$
\varphi_{q}(t)=\left(\lambda(t) t^{-1 / p_{1}}\right)^{a} t^{-1} \in Q(1,-)
$$

and thus, by Lemma 2.5(b) and a trivial estimate,

$$
\begin{gathered}
\int_{0}^{\infty}(\lambda(t))^{q} t^{-q / p_{1}}\left(\int_{t}^{\infty}\left(f^{*}(u)\right)^{q_{1}} u^{q_{1} / p_{1}} \frac{d u}{u}\right)^{q / q_{1}} \frac{d t}{t} \\
\leqslant\left(A\|f\|_{\lambda, q}^{*}\right)^{q} \leqslant B_{1}\left(\|f\|_{\lambda, q_{,}, q_{0}}^{* *}\right)^{q} .
\end{gathered}
$$

In view of the definitions of $I, \gamma$, and $\lambda_{1}$ the proof follows by making a change of variables to the integral on the left-hand side of (3.9) and by combining (3.9) $(L=K)$ with (3.10).

Proof of Theorem 2.1. In particular, the hypothesis $\lambda \in Q\left(-1 / p_{1},-1 / p_{0}\right)$ implies that, for every $k \in Z$ and $2^{k-1} \leqslant t \leqslant 2^{k}$,

$$
0<B_{0} \lambda\left(2^{k}\right) \leqslant \lambda(t) \leqslant B_{1} \lambda\left(2^{k-1}\right)
$$

and thus, for $s \geqslant q$,

$$
\begin{aligned}
\left(\|f\|_{\lambda, s}^{*}\right)^{q} & =\left(\sum_{-\infty}^{\infty} \int_{2^{k-1}}^{2^{k}}\left(\left(f^{*}(t)\right)(\lambda(t))\right)^{s} \frac{d t}{t}\right)^{q / s} \\
& \leqslant B_{2}\left(\sum_{-\infty}^{\infty}\left(\left(f^{*}\left(2^{k-1}\right)\right)\left(\lambda\left(2^{k-1}\right)\right)\right)^{s}\right)^{q / s} \\
& \leqslant B_{2} \sum_{-\infty}^{\infty}\left(\left(f^{*}\left(2^{k-1}\right)\right)\left(\lambda\left(2^{k-1}\right)\right)\right)^{q} \\
& \leqslant B_{3} \sum_{-\infty}^{\infty} \int_{2^{k-2}}^{2^{k-1}}\left(f^{*}(t)\right)^{q}(\lambda(t))^{q} \frac{d t}{t} \\
& =B_{3}\left(\|f\|_{\lambda, q}^{*}\right)^{q} .
\end{aligned}
$$

We conclude that $\|f\|_{\lambda, s}^{*} \leqslant B_{4}\|f\|_{\lambda, q}^{*}$ for $s \geqslant q$ and therefore it is sufficient to prove the theorem with $s=q$ and $q_{i}^{\prime}=\infty(i=0,1)$. 
The case $p_{1}<\infty, q<\infty$. The hypothesis $\lambda \in Q\left(-1 / p_{1},-1 / p_{0}\right)$ ensures that $\left(\lambda(t) t^{-1 / p_{0}}\right)^{a} t^{-1} \in Q(-, 1)$ and $\left(\lambda(t) t^{-1 / p_{1}}\right)^{a} t^{-1} \in Q(1,-)$ and therefore, by Lemma 2.5, the estimate (3.10) and the inequality

$$
\int_{0}^{\infty}(\lambda(t))^{q} t^{-\alpha / p_{0}}\left(\int_{0}^{l}\left(f^{*}(u)\right)^{q_{0}} u^{q_{0} / p_{0}} \frac{d u}{u}\right)^{q / q_{0}} \frac{d t}{t} \leqslant\left(A\|f\|_{\lambda, q}^{*}\right)^{q}
$$

are satisfied. Hence the proof follows by making a suitable change of variables and by applying (3.9) with $L=\infty$.

The case $p_{1}<\infty, q=\infty$. According to Lemma 2.4

$$
\begin{aligned}
(T f)^{*}(t) \cdot t^{\left(1 / p_{i}\right)-\left(1 / p_{i} \gamma\right)} \leqslant B_{5} t^{-1 / \gamma p_{0}}\left(\int_{0}^{(t / 2)^{1 / \gamma}}\left(f^{*}(u)\right)^{q_{0}} u^{q_{0} / p_{0}} \frac{d u}{u}\right)^{1 / q_{0}} \\
+t^{-1 / \gamma p_{1}}\left(\int_{(1 / 2)^{1 / \gamma}}^{\infty}\left(f^{*}(u)\right)^{q_{1}} u^{q_{1} / p_{1}} \frac{d u}{u}\right)^{1 / q_{1}} .
\end{aligned}
$$

For fixed $t>0$ we choose $k_{0} \in Z$ such that $2^{k_{0}-1} \leqslant\left(\frac{1}{2} t\right)^{1 / \gamma} \leqslant 2^{k_{0}}$. Then, by the hypothesis $\lambda \in Q\left(-,-1 / p_{0}\right)$,

$$
\begin{aligned}
\left(\int_{0}^{(u / 2)^{1 / \gamma}}\left(f^{*}(u)\right)^{q_{0}} u_{0}^{q_{0} / p_{0}} \frac{d u}{u}\right)^{1 / q_{0}} & \leqslant\|f\|_{\lambda, \infty}^{*}\left(\int_{0}^{2^{k_{0}}} u^{q_{0} / p_{0}}(\lambda(u))^{-q_{0}} \frac{d u}{u}\right)^{1 / q_{0}} \\
& \leqslant B_{6}\|f\|_{\lambda, \infty}^{*}\left(\sum_{k=-\infty}^{k-k_{0}}\left(2^{k / p_{0}} / \lambda\left(2^{k}\right)\right)^{q_{0}}\right)^{1 / q_{0}} \\
& \leqslant B_{7}\|f\|_{\lambda, \infty}^{*} 2^{k_{0} / p_{0}}\left(\lambda\left(2^{k_{0}}\right)\right)^{-1} \\
& \leqslant B_{8}\|f\|_{\lambda, \infty}^{*} t^{1 / \gamma p_{0}}\left(\lambda\left(t^{1 / \gamma}\right)\right)^{-1}
\end{aligned}
$$

Since $\lambda \in Q\left(-1 / p_{1},-\right)$ we find that

$$
\begin{aligned}
\left(\int_{(1 / 2)^{1 / \gamma}}^{\infty}\left(f^{*}(u)\right)^{q_{1}} u^{q_{1} / p_{1}} \frac{d u}{u}\right)^{1 / q_{1}} & \leqslant B_{9}\|f\|_{\lambda, \infty}^{*}\left(\sum_{k_{0}-1}^{\infty}\left(2^{k / p_{1}} / \lambda\left(2^{k}\right)\right)^{q_{1}}\right)^{1 / q_{1}} \\
& \leqslant B_{10}\|f\|_{\lambda, \infty}^{*} 2^{k_{0} / p_{1}}\left(\lambda\left(2^{k_{0}}\right)\right)^{-1} \\
& \leqslant B_{11}\|f\|_{\lambda, \infty}^{*} t^{1 / \gamma p_{1}}\left(\lambda\left(t^{1 / \gamma}\right)\right)^{-1} .
\end{aligned}
$$

We combine (3.11)-(3.13) and find that, for every $t>0$,

$$
(T f)^{*}(t) t^{\left(1 / p_{i}^{\prime}\right)-\left(1 / p_{i} \gamma\right)} \lambda\left(t^{1 / \gamma}\right) \leqslant B_{12}\|f\|_{\lambda, \infty}^{*} .
$$

Therefore, according to the definitions of $\gamma$ and $\lambda$, the theorem is proved for this case too.

The case $p_{1}=q_{1}=q=\infty$. We use the notation from the proof of Lemma 2.4 and write $f(x)=f^{\nu}(x)+f_{y}(x)$. Then by the hypothesis $(2.1)$ 
(with $i=1$ )

$$
\begin{aligned}
g_{2}^{*}(t) t^{1 / p_{1}} \lambda\left(t^{1 / \gamma}\right) & \leqslant B_{13} \lambda\left(t^{1 / \gamma}\right) \sup _{u>0} f_{y}^{*}(u) \\
& =B_{13} \lambda\left(t^{1 / \gamma}\right) f^{*}\left(t^{1 / \gamma}\right) \\
& \leqslant B_{13}\|f\|_{\lambda, \infty}^{*} .
\end{aligned}
$$

Since $\left(f^{\nu}\right)^{*}(u) \leqslant f^{*}(u)$ for $0<u<t^{1 / \gamma}$ and $\left(f^{\nu}\right)^{*}(u)=0$ for $u>t^{1 / \gamma}$ it follows from (3.13) and (2.1) (with $i=0$ ) that

$$
g_{1}^{*}(t) t^{\left(1 / p_{0}{ }^{\prime}\right)-\left(1 / p_{0} \gamma\right)} \lambda\left(t^{1 / \gamma}\right) \leqslant B_{14}\|f\|_{\lambda, \infty}^{*} \text {. }
$$

According to the definition of $\lambda_{1}$ the proof of this case follows when we combine (1.2), (3.14), and (3.15).

The case $p_{1}=q_{1}=\infty, q<\infty$. We apply (2.1) (with $i=0$ ) to the function $f^{v}(x)$ and use Lemma 2.5(a) to obtain that

$$
\int_{0}^{\infty}\left(g_{1}^{*}(t) t^{\left(1 / p_{0}{ }^{\prime}\right)-\left(1 / p_{0} \gamma\right)} \lambda\left(t^{1 / \gamma}\right)\right)^{q} \frac{d t}{t} \leqslant B_{15}\|f\|_{\lambda, q}^{*}
$$

The desired conclusion follows easily from (1.2), (3.14), and (3.16). The proof is complete.

\section{Some applications}

I. A more general version of Theorem I

We shall state a theorem which, for example, can be applied to the Fourier operators $F_{d}$ (see p. 296) and $F_{c}$ (see this page).

Theorem 4.1. Let $T$ be a quasi-linear operator of weak-type $(2 r, 2 r)$, where $r>0$, and let $\|T f\|_{\infty, \infty}^{*} \leqslant A_{0}\|f\|_{r, r}^{*}$.

(a) If $\lambda \in Q(-1 / 2 r,-)$ then, for $0<q<\infty$,

$$
\left(\int_{2 / K}^{\infty}\left((T f)^{*}(t)\right)^{q}\left(t^{1 / r} \lambda\left(t^{-1}\right)\right)^{q} \frac{d t}{t}\right)^{1 / q} \leqslant A\|f\|_{\lambda, q, r}^{* *}
$$

(b) If $\lambda \in Q(-1 / 2 r,-1 / r)$ then, for $q>0$ and $s \geqslant q$,

$$
\|T f\|_{i^{\prime / r} \lambda(1 / t), s}^{*} \leqslant A\|f\|_{\lambda, q}^{*} .
$$

The constant $A$ depends only on $\lambda, q$, and $T$.

Proof. The hypothesis asserts that the operator $T$ satisfies (2.1) with $p_{0}=q_{0}=r, p_{1}^{\prime}=p_{1}=q_{1}=2 r$, and $p_{0}^{\prime}=q_{0}^{\prime}=q_{1}^{\prime}=\infty$. Hence $\gamma=-1$ and $\lambda_{1}(t)=t^{1 / r} \lambda\left(t^{-1}\right)$ and the proof follows when we apply Theorems 2.1 and 2.3.

We note that Theorem $I$ and the analogous estimates for multiple Fourier series are special cases of Theorem 4.1 with $r=1$.

The Fourier transform, $F_{c}$, of a function $f \in L\left(\mathbf{R}^{n}\right)$ is defined by

$$
F_{c}(f)(\bar{x})=\hat{f}(\bar{x})=\int_{\mathbf{R}^{n}} f(\bar{t}) e^{-2 \pi i \bar{x} \bar{s}} d \bar{t} .
$$


The Fourier transform can also be defined for all $f \in L^{\dot{2}}\left(\mathbf{R}^{n}\right)$ and $\|f\|_{2,2}^{*}=\|\hat{f}\|_{2,2}^{*}$ (Plancherel's formula). It is easily seen that if $\lambda \in Q\left(-\frac{1}{2},-1\right)$ and if $f \in \Lambda(\lambda, q)$ then $f \in L^{1}+L^{2}$. Thus $\hat{f}$ is defined for every $f \in \Lambda(\lambda, q)$ when $\lambda \in Q\left(-\frac{1}{2},-1\right)$. We conclude that by applying Theorem 4.1(b) .with $r=1$ we find that, for every $s \geqslant q, q>0$, and $\lambda \in Q\left(-\frac{1}{2},-1\right)$,

$$
\|\hat{f}\|_{(\lambda(1 / 1), s}^{*} \leqslant B\|f\|_{\lambda, q}^{*} .
$$

For the case where $\lambda(t)=t^{1 / p}, 1<p<2\left(t \lambda\left(t^{-1}\right)=t^{1-(1 / p)}\right)$, see also Hunt $[5$, p. 270].

II. Necessary and sufficient integrability conditions of functions and their Fourier transforms

We shall now state a more general version of a theorem of Hunt [5, p. 270]. For the proof of this theorem we need the following extension of a lemma of Zygmund [17, vol. II, p. 130].

LEMMa 4.2. Assume that $f$ is an even function, which is non-negative, locally integrable, non-increasing on $[0, \infty[$ and $f(x) \rightarrow 0$ as $x \rightarrow \infty$. Furthermore, suppose that $q>0, \quad g(x)=\int_{0}^{\infty} f(s) \cos x s d s, \quad \lambda \in Q(0,-1)$, and $\lambda_{1}(t)=t \lambda(1 / t)$. Then $f \in \Lambda(\lambda, q)$ if and only if $g \in \Lambda\left(\lambda_{1}, q\right)$.

Proof. Assume that $f \in \Lambda(\lambda, q)$. We have, for every $x \in \mathbf{R}$,

$$
|g(x)| \leqslant B_{1} \int_{0}^{1 /|x|} f(s) d s \leqslant B_{1} \int_{0}^{1 /|x|} f^{*}(u) d u
$$

(see [5, p. 271]). We conclude that $g^{*}\left(t^{-1}\right) \leqslant B_{1} \int_{0}^{t} f^{*}(u) d u$ and thus

$$
\int_{0}^{\infty}\left(g^{*}\left(t^{-1}\right)\right)^{q} t^{-q}(\lambda(t))^{q} \frac{d t}{t} \leqslant B_{1}^{q} \int_{0}^{\infty}(\lambda(t))^{q} t^{-q}\left(\int_{0}^{t} f^{*}(u) d u\right)^{q} \frac{d t}{t} .
$$

We use the hypothesis $\lambda \in Q(-,-1)$ and find that

$$
\varphi_{q}(t)=\lambda((t))^{q} t^{-q-1} \in Q(-, 1) .
$$

Therefore, according to Lemma 2.5(a), the integral on the right-hand side of (4.2) is majorized by $\left(A\|f\|_{\lambda, q}^{*}\right)^{q}$. Thus we can make a change of variables in (4.2) and obtain that $\|g\|_{\lambda_{1}, q}^{*} \leqslant A B_{1}\|f\|_{\lambda, q}^{*}$.

On the other hand, suppose that $g \in \Lambda\left(\lambda_{1}, q\right)$. We have that

$$
f(t) \leqslant B_{2}\left|\int_{0}^{1 / t} g(x) d x\right|
$$

(see $[5$, p. 270] and compare with [17, vol. II, p. 129]). Therefore $f^{*}(t) \leqslant B_{2} \int_{0}^{1 / t} g^{*}(u) d u$ so that

$$
\int_{0}^{\infty}\left(f^{*}(t)\right)^{q}(\lambda(t))^{q} \frac{d t}{t} \leqslant B_{2}^{q} \int_{0}^{\infty}(\lambda(t))^{q}\left(\int_{0}^{1 / t} g^{*}(u) d u\right)^{q} \frac{d t}{t} .
$$


By hypothesis $\lambda\left(t^{-1}\right) \in Q(-, 0)$ and thus we can make a change of variables in the integral on the right-hand side in (4.3) and apply Lemma 2.5(a) with $\varphi_{q}(t)=(\lambda(t))^{q} t^{-1}$ to obtain that $\|f\|_{\lambda, q}^{*} \leqslant A B_{2}\|g\|_{\lambda_{1}, q}^{*}$. The proof is complete.

Theorem 4.3. Let $q>0$ and $\lambda_{1}(t)=t \lambda\left(t^{-1}\right)$.

(a) Assume that $\lambda \in Q\left(-\frac{1}{2},-1\right)$. Then $f \in \Lambda(\lambda, q)$ if and only if for every function $h$, such that $h^{*}=f^{*}$, the Fourier transform, $h$, exists and $\hat{h} \in \Lambda\left(\lambda_{1}, q\right)$. Moreover, $\|\hat{h}\|_{\lambda_{1}, q}^{*} \leqslant B\|f\|_{\lambda, q}^{*}$ for every such function $h$.

(b) Suppose that $\lambda \in Q\left(0,-\frac{1}{2}\right)$. Then $f \in \Lambda(\lambda, q)$ if and only if for some function $h$, such that $h^{*}=f^{*}$, the Fourier transform, $h$, exists and $\hat{h} \in \Lambda\left(\lambda_{1}, q\right)$. Furthermore, $\|f\|_{\lambda, q}^{*} \leqslant B\|\hat{h}\|_{\lambda_{1}, q}^{*}$ for every such function $h$.

Proof. Assume that $n=1$.

(a) Let $f \in \Lambda(\lambda, q)$. Then $h \in \Lambda(\lambda, q)$ and the existence of $h$ is guaranteed (see p. 304). Also, by (4.1), $\|\hat{h}\|_{\lambda_{1}, q}^{*} \leqslant B\|h\|_{\lambda, q}^{*}=B\|f\|_{\lambda, q}^{*}$. Conversely we choose $h=f^{*}$ and note that, by assumption, $h \in \Lambda\left(\lambda_{1}, q\right)$. Therefore we can use Lemma 4.2 to conclude that $h \in \Lambda(\lambda, q)$. Since $h^{*}=f^{*}$ we find that $f \in \Lambda(\lambda, q)$ and the proof of (a) is complete.

(b) Suppose that $f \in \Lambda(\lambda, q)$. Put $h=f^{*} \in \Lambda(\lambda, q)$ and use Lemma 4.2 to see that $\hat{h} \in \Lambda\left(\lambda_{1}, q\right)$. On the other hand, since $\hat{h}(x)=-h(-x)$ and $\lambda(t) \in Q\left(0,-\frac{1}{2}\right)$ if and only if $\lambda_{1}(t) \in Q\left(-\frac{1}{2},-1\right)$, we can use (4.1) to obtain that $\|f\|_{\lambda, q}^{*}=\|h\|_{\lambda, q}^{*} \leqslant B\|h\|_{\lambda_{1}, q}^{*}$. For the case where $n>1$ we study the function $h\left(x_{1}, x_{2}, \ldots, x_{n}\right)=f\left(x_{1}\right) x_{[0,1]}\left(x_{2}\right) \ldots \chi_{[0,1]}\left(x_{n}\right)$, where $f\left(x_{1}\right)$ is defined as in Lemma 4.2 and $\chi_{[0,1]}$ is the characteristic function of the interval $[0,1]$. Then the proof follows just as above.

Remark. For the case where $\lambda(t)=t^{1 / p}\left(\lambda_{1}(t)=t^{1-(1 / p)}=t^{1 / p^{\prime}}\right), p>1$, $q \geqslant 1$, see Hunt [5, p. 270].

Remark. If we study Fourier analysis when the underlying dual groups are $G=\mathbf{R}(\bmod 1)$ and $\hat{G}=\mathbf{Z}$, then the corresponding theorem has been stated by the present author in [13].

III. On operators simultaneously of weak-type $(r, r)$ and weak-type $(p, p)$ : a suggestion

We shall state a theorem, which can be seen as a refined version of the usual $L^{p}$-estimates (see, for example, $[16$, p. 21]). The theorem also improves a result of $O^{\prime}$ Neil (see $[10$, p. 464] and $[2$, p. 235]).

TheOREM 4.4. Let $T$ be a quasi-linear operator simultaneously of weaktype $(r, r)$ and weak-type $(p, p)$, where $0<r<p<\infty$.

(a) If $\lambda \in Q(-1 / p,-)$ then, for $q>0$,

$$
\left(\int_{0}^{2 K}\left((T f)^{*}(t)\right)^{q}(\lambda(t))^{q} \frac{d t}{t}\right)^{1 / q} \leqslant B\|f\|_{\lambda, q, r}^{* *}
$$


(b) If $\lambda \in Q(-1 / p,-1 / r)$, then, for every $s \geqslant q$,

$$
\|T f\|_{\lambda, s}^{*} \leqslant B\|f\|_{\lambda, q}^{*} .
$$

Proof. The hypotheses on the operator $T$ are equivalent to saying that the condition (2.1) is satisfied with $p_{0}=q_{0}=p_{0}^{\prime}=r, p_{1}=q_{1}=p_{1}^{\prime}=p$, and $q_{0}^{\prime}=q_{1}^{\prime}=\infty$. Therefore $\gamma=1$ and $\lambda_{1}(t)=\lambda(t)$, so the proof follows when we apply Theorems 2.1 and 2.3 to the considered operator $T$.

We note that if $\lambda(t)=t^{1 / a}$, where $r<a<p$, then $\lambda \in Q(-1 / p,-1 / r)$ and (4.4) is turned into the estimate $\|T f\|_{a, 8}^{*} \leqslant B\|f\|_{a, q}^{*}$. For the case where $s=q=a$ and $r=1$ see $[16$, p. 21]. Moreover, Theorem 4.4(a) contains the fact that if $f \in \Lambda\left(t^{1 / a}(\log (2+t))^{b}, q\right)$, for $r<a<p, b \in \mathbf{R}$, then, for $s \geqslant q$, $T f \in \Lambda\left(t^{1 / a}(\log (2+t))^{b}, s\right)$ for every operator of the type considered. We also observe that, by applying Theorem 4.4(a), with $K=1$ and $\lambda(t)=t^{1 / r}\left(\log t^{-1}\right)^{b-(1 / q)}$, where $1 \leqslant q \leqslant \infty$, we obtain the inequality

$$
\int_{0}^{1}\left((T f)^{*}(t)\right)^{q} t^{q / r}\left(\log t^{-1}\right)^{b q-1} \frac{d t}{t} \leqslant B \int_{0}^{1}\left(\log t^{-1}\right)^{b q-1}\left(\int_{0}^{t}\left(f^{*}(u)\right)^{r} d u\right)^{q / r} \frac{d t}{t}
$$

for every operator $T$ which is of weak-type $(r, r)$ and weak-type $(p, p)$. In particular, for the case where $q=r$ we can make a partial summation and use a standard argument (see p. 307) to see that

$$
\int_{0}^{1}|T f(x)|^{r}\left(\log ^{+}|T f(x)|\right)^{b r-1} d x \leqslant B \int_{0}^{1}|f(x)|^{r}\left(\log ^{+}|f(x)|\right)^{b r} d x+B .
$$

Therefore it is tempting to suggest that if the quasi-linear operator $T$ is of weak-type $(r, r)$ and if

$$
T: L r(\log +L)^{b_{0} r} \rightarrow L^{r}(\log L)^{b_{0} r-1},
$$

where $b_{0} \geqslant 0$, then

$$
T: \Lambda_{A}\left(t^{1 / r}\left(\log t^{-1}\right)^{b-(1 / q)}, q, r\right) \rightarrow \Lambda\left(t^{1 / r}\left(\log t^{-1}\right)^{b-(1 / q)}, q\right)
$$

where $b_{0}-1<b<b_{0}, q>0$. According to a recent result of Bennett (see [2, p. 934] and [3, p. 215]) the suggestion is correct at least when $r=1$, $b_{0}=1$, and $q \geqslant 1$. Bennett's proof depends crucially upon the fact that $\Lambda(X)=X$ for the spaces $L$ and $L \log ^{+} L(\Lambda(X)$ denotes the $\Lambda$-space associated with $X$, see, for example, $[3$, p. 220]). Unfortunately, the spaces $L^{r} \log ^{+} L$, where $r>1$, for example, are not $\Lambda$-spaces (see [9, p. 127]) so the technique developed by Bennett cannot be extended to the case where $r>1$.

IV. Summability relations between functions and their maximal and conjugate functions

For the sake of convenience we shall assume that $f \in L[0,1]$. As usual, $M f$ stands for the Hardy-Littlewood maximal function of $f$ (see [17, 
vol. I, p. 30]). The conjugate function of $f$ is denoted $f$ (see $[17$, vol. I, p. 131]). It is easy to verify that

$$
f^{* *}(2 x) \leqslant M\left(f^{*}\right)(x) \leqslant f^{* *}(x) \quad(0<x \leqslant 1),
$$

where $f^{* *}(x)=x^{-1} \int_{0}^{x} f^{*}(u) d u$ (see [3, p. 218]). The following nice estimate is due to Herz (see [4]):

$$
f^{* *}(2 x) \leqslant 6(M f)^{*}(2 x) \leqslant 24 f^{* *}(x) \quad\left(0<x \leqslant \frac{1}{2}\right) .
$$

It is well known that the mapping $f \rightarrow f$ is of weak-type $(1,1)$ and of (weak-) type $(p, p)$ for all $p>1$ (see, for example, [6, pp. 66-68]). Therefore, by Theorem 4.2,

$$
\|\tilde{f}\|_{\lambda, q}^{*} \leqslant B\|f\|_{\lambda, q}^{*}
$$

for every $\lambda \in Q(0,-1)$ and $q>0$. Since $\tilde{\tilde{f}}(x)=-f(x)$ a.e. (see, for example, [6, p. 63]) we can combine (4.5)-(4.7) and use Lemma 2.5(a) to obtain the following theorem:

TheORem 4.5. Let $q>0$, let $\lambda \in Q(0,-1)$, and let $h$ be any of the functions $f$ or $f$. Then the following conditions are equivalent:

$$
\begin{gathered}
h \in \Lambda(\lambda, q), \quad h \in \Lambda_{\Delta}(\lambda, q, 1), \quad M h \in \Lambda(\lambda, q), \\
M h \in \Lambda_{\Delta}(\lambda, q, 1), \quad M h^{*} \in \Lambda(\lambda, q), \quad \text { and } \quad M h^{*} \in \Lambda_{\Delta}(\lambda, q, 1) .
\end{gathered}
$$

Theorem 4.5 is false, for example, when $\lambda(t)=t\left(\log t^{-1}\right)^{\theta}$, where $\theta>-1$ (see [17, vol. I, Chapter 5]). This observation depends on the fact that the estimate (4.7) does not hold for this case. (However, the estimate $\|f\|_{\lambda, q}^{*} \leqslant B\|f\|_{\lambda, q, 1}^{* *}$ holds for every $\lambda \in Q(0,-)$.)

Finally we shall only state the following generalization of a recent result of Bennett (see [3, p. 218]).

Theorem 4.6. If $\varphi\left(2^{b}\right) \in Q(-,-)$, if $\varphi_{1}\left(2^{b}\right)=\int_{0}^{t} \varphi\left(2^{x}\right) d x$, and if $f \in L[0,1]$, then the following conditions are equivalent:

and

$$
\begin{array}{cl}
f \in \Lambda\left(t \varphi_{1}(t), 1\right), & f \in \Lambda_{A}(t \varphi(t), 1,1), \quad f \in L \varphi_{1}(L), \\
M f \in \Lambda(t \varphi(t), 1), & M f \in L \varphi(L), \quad M f^{*} \in \Lambda(t \varphi(t), 1), \\
& M f^{*} \in L \varphi(L) .
\end{array}
$$

Proof. The proof follows easily by applying (4.5) and (4.6), by making partial summations, and by using the fact that we may, without loss of generality, assume that $u^{-t} \leqslant f^{*}(u) \leqslant u^{-1}$ and thus $\varphi(t) \simeq \varphi\left(f^{*}\right)$ and $\varphi_{1}(t) \simeq \varphi_{1}\left(f^{*}\right)($ see $[11])$.

For example, if $\varphi(t)=\left(\log ^{+} t^{-1}\right)^{\theta}$, where $\theta \geqslant-1$, then $\left.\varphi_{1}(t)=\log ^{+} t^{-1}\right)^{\theta+1}$ when $\theta>-1$ and $\varphi_{1}(t)=\log ^{+} \log ^{+} t^{-1}$ when $\theta=-1$. Compare with the work of Bennett [3, p. 218] and Stein [15, p. 305]. 


\title{
REFERENCES
}

1. C. BenNeTr, 'Banach function spaces and interpolation methods III. HausdorffYoung estimates', J. Approximation Theory, to appear.

2. _Estimates for weak-type operators', Bull. Amer. Math. Soc. 79 (1973) 933-35.

3. - 'Intermediate spaces and the class $L \log ^{+} L^{\prime}$ ', Ark. Mat. 11 (1973) 216-28.

4. C. S. Herz, 'The Hardy-Littlewood maximal theorem', in Symposium in harmonic anaylsis (Wiley, New York, 1968).

5. R. A. HUNT, 'On $L(p, q)$ spaces', Enseignement Math. 12 (1966) 249-76.

6. Y. Katznelson, An introduction to harmonic analysis (Wiley, Now York, 1968).

7. L. LEINDLER, 'Generalization of inequalities of Hardy and Littlewood', Acta Sci. Math. (1970) 279-85.

8. G. G. LoRentz, 'Some new functional spaces', Ann. of Math. 51 (1950) 37-55.

9. _Relations between function spaces', Proc. Amer. Math. Soc. 12 (1961) 127-32.

10. R. O'Nerr, 'Les fonctions conjugées et les intégrales fractionnaires de la classe $L\left(\log ^{+} L\right)^{\circ}, C$. R. Acad. Sci. Paris Sér. A-B 263 (1966) 463-66.

11. L.-E. Persson, 'Relations between summability of functions and their Fourier series', Acta Sci. Math. 27 (1976) 267-80.

12. - 'Relations between regularity of periodic functions and their Fourier series', Research report, Department of Mathematics, University of Umea, No. 3 (1974).

13. - Relations between regularity of periodic functions and their Fourier series', Research report, Department of Mathematics, University of Umea,, No. 4 (1974) (thesis).

14. E. M. SterN, 'Interpolation of linear operators', Trans. Amer. Math. Soc. 83 (1956) 482-92.

15. - 'Note on the class $L \log ^{+} L$ ', Studia Math. 32 (1969) 305-10.

16. - Singular integrals and differentiability properties of functions (Princeton University Press, Princeton, N.J., 1970).

17. A. ZYGMUND, Trigonometric series, Vols I and II, 2nd revised edn (Cambridge University Press, New York, 1959).

\author{
Section of Mathematics \\ Department of Machine Engineering \\ University of Lulea \\ 95187 Luled, Sweden \\ and \\ University of Umed \\ S-901 87 Umed, Sweden
}

